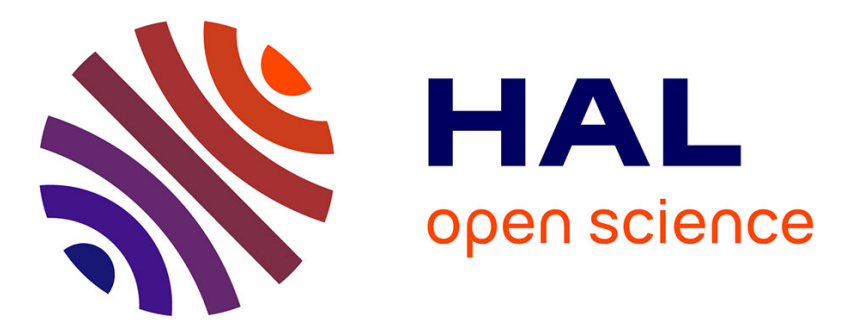

\title{
Robust Feedback Stabilization of Linear MIMO Systems Using Generalized Homogenization
}

\author{
Konstantin Zimenko, Andrey Polyakov, Denis Efimov, Wilfrid Perruquetti
}

\section{To cite this version:}

Konstantin Zimenko, Andrey Polyakov, Denis Efimov, Wilfrid Perruquetti. Robust Feedback Stabilization of Linear MIMO Systems Using Generalized Homogenization. IEEE Transactions on Automatic Control, In press, 10.1109/TAC.2020.2969718 . hal-02614543

\section{HAL Id: hal-02614543 \\ https://hal.inria.fr/hal-02614543}

Submitted on 21 May 2020

HAL is a multi-disciplinary open access archive for the deposit and dissemination of scientific research documents, whether they are published or not. The documents may come from teaching and research institutions in France or abroad, or from public or private research centers.
L'archive ouverte pluridisciplinaire HAL, est destinée au dépôt et à la diffusion de documents scientifiques de niveau recherche, publiés ou non, émanant des établissements d'enseignement et de recherche français ou étrangers, des laboratoires publics ou privés. 


\title{
Robust Feedback Stabilization of Linear MIMO Systems Using Generalized Homogenization*
}

\author{
Konstantin Zimenko, Andrey Polyakov, Denis Efimov and Wilfrid Perruquetti
}

\begin{abstract}
A robust nonlinear control is designed for stabilizing linear MIMO systems. The presented control law homogenizes a linear system (without its transformation to a canonical form) with a specified degree and stabilizes it in a finite time (or with a fixed-time attraction to any compact set containing the origin) if the degree of homogeneity is negative (positive). The tuning procedure is formalized in LMI form. Performance of the approach is illustrated by numerical and experimental examples.
\end{abstract}

Index Terms-Generalized homogeneity, finite-time stabilization, linear MIMO systems.

\section{INTRODUCTION}

$\mathbf{H}$ OMOGENEITY is a dilation symmetry, which is widely used in control theory for system analysis, regulation and observer design (see, for example, [5]-[22]). Such an interest to the homogeneity concept is based on various useful features of homogeneous systems. In particular, a local stability of homogeneous system means the global one; the rate of convergence of homogeneous systems can be assessed by its homogeneity degree; homogeneous systems are robust with respect to external perturbations and time delays.

The standard homogeneity means a mathematical object $f$ (for example, function or vector field) remains invariant with respect to scaling of its argument $f\left(e^{s} x\right)=e^{\nu s} f(x), s, \nu \in \mathbb{R}$. In the general case, instead of the uniform scaling one can consider a non-uniform scaling $x \rightarrow \mathbf{d}(s) x$, where an operator $\mathbf{d}(s): \mathbb{R}^{n} \rightarrow \mathbb{R}^{n}$ is called dilation if $\|\mathbf{d}(s) x\| \rightarrow 0$ as $s \rightarrow$ $-\infty$ and $\|\mathbf{d}(s) x\| \rightarrow+\infty$ as $s \rightarrow+\infty$ for $x \neq 0$. In [2], [3], [4], the dilation $\mathbf{d}$ is suggested to be generated as a flow of $C^{1}$ vector field. Such a dilation is known as geometric dilation. The present paper deals with one of generalized homogeneity concepts [5], [6], which is based on groups of linear dilations. In the context of the results [2], [3] this type of homogeneity can be called linear geometric homogeneity.

The present paper addresses two problems. First, feedback homogenization, i.e. problem of a feedback design making the

*The material in this paper was partially presented at the 17th annual European Control Conference, Limassol, Cyprus [1]. The results on homogenization of linear MIMO systems presented in Section V were developed under support of RSF (grant 17-19-01422) in ITMO University. The other research were partially supported by ANR 15 CE23 0007 (Project Finite4SoS) and Goverment of Russian Federation (Grant 08-08).

Konstantin Zimenko, Andrey Polyakov and Denis Efimov are with Faculty of Control Systems and Robotics, ITMO University, 49 Kronverkskiy av., 197101 Saint Petersburg, Russia. (e-mail: kostyazimenko@gmail.com).

$\begin{gathered}\text { Andrey } \\ \text { ruquetti } \\ \text { are with }\end{gathered}$ Inria, Univ. Lille, CNRS, UMR
9189 - CRIStAL, F-59000 Lille, France. (e-mail:
denis.efimoveinria.fr, andrey.polyakove
inria.fr, Wilfrid.Perruquettidinria.fr).

system homogeneous of a given degree. Second, homogeneous stabilization of linear MIMO plants. The presented homogeneous feedbacks have several advantages:

- the closed-loop system is robust in the sense of Input-toState Stability (ISS);

- it allows to reject some non-Lipschitz disturbances in the case of non-zero homogeneity degree;

- the origin is finite-time stable (every compact set containing the origin is fixed-time attractive) in the case of negative (positive) homogeneity degree.

Moreover, unlike existing solutions on homogeneous control design, the presented results do not require special canonical forms of the system matrix or block decomposition of linear MIMO model (as, for example, in [7]), which can be accompanied by significant computational errors.

In comparison with the conference version [1], in addition to detailed proofs, the presented results allow to derive necessary conditions of generalized homogenizability and homogeneous stabilizability of linear MIMO systems. Moreover, it is shown that if a system is homogeneously stabilizable of nonzero degree then it is homogeneously stabilizable with any degree.

The presented control law contains two terms: a linear feedback that homogenizes the system with a specified degree and a generalized homogeneous control law, which stabilizes the system in a finite time (with a fixed-time attraction of any compact set containing the origin) if the degree of homogeneity is negative (positive).

Notation: $\mathbb{R}_{+}=\{x \in \mathbb{R}: x>0\}$, where $\mathbb{R}$ is the field of real numbers; $\|\cdot\|$ denotes a norm in $\mathbb{R}^{n} ;\|A\|=\sup _{x \in \mathbb{R}^{n}} \frac{\|A x\|}{\|x\|}$ for $A \in \mathbb{R}^{n \times n} ; I_{n} \in \mathbb{R}^{n \times n}$ is the identity matrix; the eigenvalues of a matrix $G \in \mathbb{R}^{n \times n}$ are denoted by $\lambda_{i}(G)$, $i=1, \ldots, n, \lambda_{\min }(G)=\min _{i=1, \ldots,} \lambda_{i}(G)$ and $\lambda_{\max }(G)=$ $\max _{i=1, \ldots,} \lambda_{i}(G)$ if $G$ is symmetric; the inequality $P>0$ means that a symmetric matrix $P=P^{T} \in \mathbb{R}^{n \times n}$ is positive definite; $\mathfrak{R}(\lambda)$ denotes the real part of a complex number $\lambda$.

\section{PRELIMINARIES}

\section{A. Stability Notions}

Consider the following system

$$
\dot{x}(t)=f(x(t)), \quad x(0)=x_{0}, \quad t \geq 0,
$$

where $x(t) \in \mathbb{R}^{n}$ is the state vector, $f \in \mathbb{R}^{n} \rightarrow \mathbb{R}^{n}$ is continuous, $f(0)=0$.

Definition 1 [8], [9] The origin of (1) is said to be globally finite-time stable if it is globally asymptotically stable and any solution $x\left(t, x_{0}\right)$ of the system (1) reaches the equilibrium point at some finite time moment, i.e. $x\left(t, x_{0}\right)=0 \forall t \geq T\left(x_{0}\right)$ 
and $x\left(t, x_{0}\right) \neq 0 \forall t \in\left[0, T\left(x_{0}\right)\right), x_{0} \neq 0$, where $T: \mathbb{R}^{n} \rightarrow$ $\mathbb{R}_{+} \cup\{0\}, T(0)=0$ is a settling-time function.

Definition 2 [23] A set $M \subset \mathbb{R}^{n}$ is said to be globally finitetime attractive for (1) if any solution $x\left(t, x_{0}\right)$ of (1) reaches $M$ in some finite time moment $t=T\left(x_{0}\right)$ and remains there $\forall t \geq T\left(x_{0}\right), T: \mathbb{R}^{n} \rightarrow \mathbb{R}_{+} \cup\{0\}$ is a settling-time function. It is fixed-time attractive if in addition a settling-time function $T$ is globally bounded by some number $T_{\max }>0$.

Theorem 1 [8], [10] Suppose there exist a positive definite $C^{1}$ function $V$ defined on an open neighborhood of the origin $D \subset \mathbb{R}^{n}$ and real numbers $C>0$ and $\sigma \geq 0$, such that the following condition is true for the system (1)

$$
\dot{V}(x) \leq-C V^{\sigma}(x), \quad x \in D \backslash\{0\} .
$$

Then depending on the value $\sigma$ the origin is stable with different types of convergence:

- if $\sigma=1$, the origin is asymptotically stable;

- if $0 \leq \sigma<1$, the origin is finite-time stable and $T\left(x_{0}\right) \leq$ $\frac{1}{C(1-\sigma)} V_{0}^{1-\sigma}$, where $V_{0}=V\left(x_{0}\right)$;

- if $\sigma>1$ the origin is asymptotically stable and, for every $\varepsilon \in \mathbb{R}_{+}$, the set $B=\{x \in D: V(x)<\varepsilon\}$ is fixed-time attractive with $T_{\max }=\frac{1}{C(\sigma-1) \varepsilon^{\sigma-1}}$.

If $D=\mathbb{R}^{n}$ and function $V$ is radially unbounded, then the system (1) admits these properties globally.

\section{B. Generalized Homogeneity}

The homogeneity is a symmetry property of an object with respect to a group of transformations (dilation operation). The considered type of homogeneity deals with commutative linear transformations.

Definition 3 [5], [11] A map $\mathbf{d}: \mathbb{R} \rightarrow \mathbb{R}^{n \times n}$ is called dilation in the space $\mathbb{R}^{n}$ if it satisfies:

- group property: $\mathbf{d}(0)=I_{n}$ and $\mathbf{d}(t+s)=\mathbf{d}(t) \mathbf{d}(s)=$ $\mathbf{d}(s) \mathbf{d}(t)$ for $t, s \in \mathbb{R}$

- continuity property: $\mathbf{d}$ is a continuous map;

- limit property: $\lim _{s \rightarrow-\infty}\|\mathbf{d}(s) x\|=0$ and $\lim _{s \rightarrow+\infty}\|\mathbf{d}(s) x\|=+\infty$ uniformly on the unit sphere $\mathbb{S}=\left\{x \in \mathbb{R}^{n}:\|x\|=1\right\}$.

It follows from Definition 3 that the dilation $\mathbf{d}$ is a uniformly continuous group, for which there always exists a generator $G_{\mathbf{d}} \in \mathbb{R}^{n \times n}$ defined by $G_{\mathbf{d}}=\lim _{s \rightarrow 0} \frac{\mathbf{d}(s)-I_{n}}{s}$ [24]. The generator $G_{\mathbf{d}}$ satisfies the following properties [24]

$$
\begin{gathered}
\frac{d}{d s} \mathbf{d}(s)=G_{\mathbf{d}} \mathbf{d}(s)=\mathbf{d}(s) G_{\mathbf{d}}, \\
\mathbf{d}(s)=e^{G_{\mathbf{d}} s}=\sum_{i=0}^{+\infty} \frac{s^{i} G_{\mathbf{d}}^{i}}{i !}, s \in \mathbb{R} .
\end{gathered}
$$

Definition 4 [5] The dilation $\mathrm{d}$ is said to be strictly monotone if $\exists \beta>0:\|\mathbf{d}(s)\|<e^{\beta s}$ for $s \leq 0$.

Thus, monotonicity means that $\mathbf{d}(s)$ is a strong contraction for $s<0$ (strong expansion for $s>0$ ) and implies that for any $x \in \mathbb{R} \backslash\{0\}$ there exists a unique pair $\left(s_{0}, x_{0}\right) \in \mathbb{R} \times S$ such that $x=\mathbf{d}\left(s_{0}\right) x_{0}$.

Theorem 2 [6] If $\mathbf{d}$ is a dilation in $\mathbb{R}^{n}$, then

- the generator matrix $G_{\mathbf{d}}$ is anti-Hurwitz (i.e. $\mathfrak{R}\left(\lambda_{i}\left(G_{\mathbf{d}}\right)\right)>$ $0, i=1, \ldots, n)$ and there exists a matrix $P \in \mathbb{R}^{n \times n}$ such that

$$
P G_{\mathbf{d}}+G_{\mathbf{d}}^{T} P>0, \quad P>0 .
$$

- the dilation $\mathbf{d}$ is strictly monotone with respect to the norm $\|x\|=\sqrt{x^{T} P x}$ for $x \in \mathbb{R}^{n}$ and $P$ satisfying (2):

$$
\begin{aligned}
& e^{\alpha s} \leq\|\mathbf{d}(s)\| \leq e^{\beta s} \quad \text { if } \quad s \leq 0, \\
& e^{\beta s} \leq\|\mathbf{d}(s)\| \leq e^{\alpha s} \quad \text { if } \quad s \geq 0,
\end{aligned}
$$

where $\alpha=\frac{1}{2} \lambda_{\max }\left(P^{\frac{1}{2}} G_{\mathbf{d}} P^{-\frac{1}{2}}+P^{-\frac{1}{2}} G_{\mathbf{d}}^{T} P^{\frac{1}{2}}\right), \beta=$ $\frac{1}{2} \lambda_{\min }\left(P^{\frac{1}{2}} G_{\mathbf{d}} P^{-\frac{1}{2}}+P^{-\frac{1}{2}} G_{\mathbf{d}}^{T} P^{\frac{1}{2}}\right)$.

Definition 5 [5] A vector field $f: \mathbb{R}^{n} \rightarrow \mathbb{R}^{n}$ (a function $\left.g: \mathbb{R}^{n} \rightarrow \mathbb{R}\right)$ is said to be $\mathbf{d}$-homogeneous of degree $\nu \in \mathbb{R}$ if

$$
\begin{array}{cll}
f(\mathbf{d}(s) x)=e^{\nu s} \mathbf{d}(s) f(x), & \forall x \in \mathbb{R}^{n}, & \forall s \in \mathbb{R} . \\
\left(\operatorname{resp} . g(\mathbf{d}(s) x)=e^{\nu s} g(x),\right. & \forall x \in \mathbb{R}^{n}, & \forall s \in \mathbb{R} .)
\end{array}
$$

Remark 1 If $n=1$, then the generator $G_{\mathbf{d}} \in \mathbb{R}_{+}$is a positive scalar and $\mathbf{d}(s)=e^{s G_{\mathbf{d}}} \in \mathbb{R}_{+}$for any $s \in \mathbb{R}$. In this case, homogeneous vector fields/functions $\mathbb{R} \rightarrow \mathbb{R}$ satisfy both conditions in (3), but its degree can be defined differently following the context (e.g., if it defines the right-hand side of ODE, then the second formula of (3) is utilized).

Note that the homogeneity studied in this paper was introduced in [5] for analysis of partial differential equations, it is more general than the weighted homogeneity and less general than the geometric one. Indeed, in the case of geometric homogeneity, the dilation group may be nonlinear. In this context, the generalized homogeneity studied in the paper is classified as a linear geometric homogeneity.

A special case of homogeneous function is a homogeneous norm [6], [2]: a continuous positive definite d-homogeneous function of degree 1. For monotone dilations we define the canonical homogeneous norm $\|\cdot\|_{\mathbf{d}}: \mathbb{R}^{n} \rightarrow \mathbb{R}_{+} \cup\{0\}$ as $\|x\|_{\mathbf{d}}=e^{s_{x}}$ for $x \neq 0$, where $s_{x} \in \mathbb{R}$ such that $\left\|\mathbf{d}\left(-s_{x}\right) x\right\|=1$ and, by continuity, we assign $\|0\|_{\mathbf{d}}=0$. Note that $\|\mathbf{d}(s) x\|_{\mathbf{d}}=e^{s}\|x\|_{\mathbf{d}}$ and

$$
\left\|\mathbf{d}\left(-\ln \|x\|_{\mathbf{d}}\right) x\right\|=1 .
$$

If the dilation $\mathbf{d}$ is monotone then the function $\|\cdot\|_{\mathbf{d}}$ is continuous on $\mathbb{R}^{n}$ and Lipschitz continuous on $\mathbb{R}^{n} \backslash\{0\}$ (see [6]).

Theorem 3 [6] An asymptotically stable d-homogeneous system $\dot{x}=f(x), f: \mathbb{R}^{n} \rightarrow \mathbb{R}^{n}$ of degree $\nu \in \mathbb{R}$ is uniformly finite-time stable if and only if $\nu<0$.

\section{On the Matrix Equation $X \bar{A}-\bar{A} X=X$}

Let us consider the matrix equation

$$
X \bar{A}-\bar{A} X=X
$$

Lemma 1 [25] Let $\bar{A} \in \mathbb{R}^{n \times n}$. Then every matrix solution $X \in \mathbb{R}^{n \times n}$ of equation (5) is nilpotent.

Note, that the equation (5) is a special case of the Sylvester matrix equation. The general solution of the matrix equation is given in [26]. In solving the inverse problem (search for the matrix $\bar{A}$ for given $X$ ), the following result is valid.

Lemma 2 For any nilpotent matrix $X \in \mathbb{R}^{n \times n}$ equation (5) has a solution.

Proofs of all theorems and lemmas are given in the Appendix. 


\section{PROBLEM Formulation}

Firstly, introduce the notions of generalized homogenization and generalized homogeneous stabilization.

A nonlinear system $\dot{x}=f(x, u)$ is

- d-homogenizable with degree $\nu \in \mathbb{R}$ if there exists a feedback $u(x)$ such that the closed-loop system is $\mathbf{d}$ homogeneous of degree $\nu$.

- d-homogeneously stabilizable with degree $\nu \in \mathbb{R}$ if there exists a feedback $u(x)$ such that the closed-loop system is $\mathbf{d}$ homogeneous of degree $\nu$ and globally asymptotically stable.

This paper studies the problem of generalized homogenization and robust stabilization for the linear plant

$$
\dot{x}(t)=A x(t)+B u(t)+\omega(t, x(t)),
$$

where $x \in \mathbb{R}^{n}$ is the state vector, $u \in \mathbb{R}^{m}$ is the vector of control inputs, $A \in \mathbb{R}^{n \times n}, B \in \mathbb{R}^{n \times m}$ (the pair $(A, B)$ is controllable, rank $\mathrm{B}=m \leq n$ ) are system and control gain matrices, $\omega: \mathbb{R} \times \mathbb{R}^{n} \rightarrow \mathbb{R}^{n}$ models the system uncertainties and disturbances. The state vector $x$ is assumed to be measured. The case of discontinuous function $\omega$ is treated using the theory of Filippov [27].

The main goal is to propose a constructive (i.e. equipped with reliable tuning rules and robustness abilities) stabilizing control algorithm based on generalized homogenization of the system (6). Notice that in the case of negative (positive) homogeneity degree the control guarantees finite-time stability (asymptotic stability with a fixed-time attraction of any compact set containing the origin).

\section{MAIN RESULT}

Let us rewrite the result of [2] for generalized homogeneity case that gives the criterion for a linear system to be $\mathbf{d}$ homogeneous with the generator $G_{\mathbf{d}}$.

Lemma 3 Let $G_{\mathbf{d}} \in \mathbb{R}^{n \times n}$ be a generator of the dilation $\mathbf{d}(s)=e^{G_{\mathbf{d}} s}, s \in \mathbb{R}$. Then the linear system $\dot{x}=C x, x \in \mathbb{R}^{n}$, $C \in \mathbb{R}^{n \times n}$ is d-homogeneous of degree $\nu \in \mathbb{R}$ if and only if

$$
C G_{\mathbf{d}}-G_{\mathbf{d}} C=\nu C \text {. }
$$

Based on Lemma 1 and Lemma 3 it can be shown that the system (6) with $\omega(t, x)=0$ can be homogenized by a linear control $u=K_{0} x$.

Theorem 4 (Disturbance-free case) The disturbance-free closed-loop system (6) with $u=K_{0} x, K_{0} \in \mathbb{R}^{m \times n}$ is dhomogeneous of degree $\nu \neq 0$ for some dilation $\mathbf{d}$ if and only if $A+B K_{0}$ is a nilpotent matrix.

Therefore, the control $u=K_{0} x$, where $K_{0}$ is such that $A+B K_{0}$ is a nilpotent matrix, homogenizes the system (6) with any degree $\nu \in \mathbb{R}$ (for a dilation dependent on $\nu$ and $K_{0}$ ). Further, these results are utilized for control design of the system (6) with $u=K_{0} x+\tilde{u}$, where $\tilde{u}$ is d-homogeneous, then we have the following property:

Lemma 4 If the disturbance-free system (6) is $\mathbf{d}$ homogeneously stabilizable of degree $\nu \neq 0$ then it is $\mathbf{d}$ homogeneously stabilizable with any degree.

The following theorem gives the necessary and sufficient condition for $\mathbf{d}$-homogeneous stabilizability of the system (6) with $\omega(t, x)=0$.
Theorem 5 (Disturbance-free case) The disturbance-free closed-loop system (6) is $\mathbf{d}$-homogeneously stabilizable with degree $\nu \neq 0$ if and only if the pair $\{A, B\}$ is controllable.

The following lemma gives the corresponding homogeneitybased stabilizing feedback law for the disturbance-free system (6).

Lemma 5 Let the control be chosen in the form

$$
u(x)=K_{0} x+\|x\|_{\mathbf{d}}^{\nu(1+\gamma)+\epsilon} K \mathbf{d}\left(-\ln \|x\|_{\mathbf{d}}\right) x,
$$

where

- $K_{0}=y_{0}\left(L-(\gamma+1) I_{n}\right)^{-1}$ for some $L \in \mathbb{R}^{n \times n}, y_{0} \in$ $\mathbb{R}^{m \times n}, \gamma \in \mathbb{R}$ satisfying

$$
\begin{gathered}
A L-L A-A+B y_{0}=0, \\
\left(L-\gamma I_{n}\right) B=0, \\
L-(\gamma+1) I_{n}<0,
\end{gathered}
$$

- $K \in \mathbb{R}^{m \times n}, \epsilon \in \mathbb{R}_{+}$are chosen such that

$$
\begin{gathered}
\left(A+B K_{0}\right) X+X\left(A+B K_{0}\right)^{T}+B y+y^{T} B^{T}+\delta X \leq 0, \\
X>0 \\
\eta X \geq \nu L X+\nu X L^{T}+2 \epsilon X>0
\end{gathered}
$$

for some $X \in \mathbb{R}^{n \times n}, y \in \mathbb{R}^{m \times n}, \delta, \eta \in \mathbb{R}_{+}$with $K=y P$, $P=X^{-1}$ and $G_{\mathbf{d}}=\nu L+\epsilon I_{n}$.

Then the disturbance-free closed-loop system (6), (8) is asymptotically (for $\nu>0) /$ exponentially (for $\nu=0) /$ finite-time (for $\nu<0$ ) stable.

Remark 2 The presented control is an extension of the results in [7], where the weighted homogeneity is used (the special case of generalized homogeneity with diagonal matrix $G_{\mathbf{d}}$ ). Indeed, applying the presented approach for the system in the canonical block form one obtains a control with the same structure as in the paper [7]. Thus the main advantage of this paper is in the fact the presented approach does not require block decomposition which in some cases may be accompanied by significant computational errors.

The presented control scheme provides different types of convergence depending on homogeneity degree $\nu$. For $\nu<0$ the closed-loop system (6), (8) is finite-time stable with the following settling time estimate $T\left(x_{0}\right) \leq-\frac{\eta}{\delta \nu}\left\|x_{0}\right\|_{\mathbf{d}}^{-\nu}$. For $\nu>0$ the closed-loop system is asymptotically stable and for any $\varepsilon \in \mathbb{R}_{+}$, the set $B=\left\{x \in \mathbb{R}^{n}: V(x)<\varepsilon\right\}$ is fixed-time attractive with $T_{\max }=\frac{\eta}{\delta \nu} \varepsilon^{-\nu}$. In the case $\nu=0$ the control scheme reduces to the standard linear control design problem.

Note that the canonical homogeneous norm $\|\cdot\|_{\mathbf{d}}$ is defined implicitly by (4). Therefore, to realize the control (8) the numerical procedures can be used to find $\|\cdot\|_{\mathbf{d}}$. For example, the bisection method may be utilized (see, e.g. [7]).

Homogeneity of the closed-loop system implies robust properties, such as, ISS stability (see, e.g., [12], [13], [14]) and non-Lipschitz disturbances rejection. The following theorem presents the modification of the control scheme to reject some additive disturbances. 
Theorem 6 (Disturbed case) Let $\nu \in \mathbb{R}$ and

- the system (9), (10b) - (10c),

$\left(A+B K_{0}\right) X+X\left(A+B K_{0}\right)^{T}+B y+y^{T} B^{T}+\delta X+R \leq 0$,

is feasible for some $K_{0} \in \mathbb{R}^{m \times n}, R, L, X \in \mathbb{R}^{n \times n}, R>0$, $y \in \mathbb{R}^{m \times n}, \delta \in \mathbb{R}_{+}, \gamma \in \mathbb{R}$;

- the control has the form (8) with $K=y P, P=X^{-1}$;

- the disturbance function $\omega$ satisfy

$\omega^{T} \mathbf{d}^{T}\left(-\ln \|x\|_{\mathbf{d}}\right) R^{-1} \mathbf{d}\left(-\ln \|x\|_{\mathbf{d}}\right) \omega \leq \zeta \delta\|x\|_{\mathbf{d}}^{2 \nu}, \zeta \in(0,1)$.

Then the system (6), (8) is asymptotically (for $\nu>0$ ) / exponentially (for $\nu=0) /$ finite-time (for $\nu<0)$ stable.

For $\nu<0$ the settling time is bounded by $T\left(x_{0}\right) \leq$ $-\frac{\eta}{(1-\zeta) \delta \nu}\left\|x_{0}\right\|_{\mathbf{d}}^{-\nu}$. For $\nu>0$ and any $\varepsilon \in \mathbb{R}_{+}$, the set $B=\left\{x \in \mathbb{R}^{n}: V(x)<\varepsilon\right\}$ is fixed-time attractive with $T_{\max }=\frac{\eta}{(1-\zeta) \delta \nu} \varepsilon^{-\nu}$.

Remark $\mathbf{3}$ Theorem 6 is proven under assumption that $\omega$ is continuous function of state. Using Filippov Theory and convex embedding technique it can be proven for discontinuous $\omega$ in the similar way. In the latter case the inequality (12) is not needed to be checked at the discontinuity set.

According to the restriction on the system disturbances (12) the control (8) allows to reject non-Lipschitz disturbances in the case of non-zero homogeneity degree. Also note that the result does not require the disturbance to be matched. The restriction (12) is presented in the form, which is not appropriate for using in practice. A more constructive (but also more conservative) condition can be derived in the following way.

Corollary 1 Let $X \in \mathbb{R}^{n \times n}$ be a solution of the system (9), $(10 b)-(11)$,

$$
X \leq \vartheta R, \vartheta \in \mathbb{R}_{+}
$$

with $P=X^{-1}$. If

$$
\sqrt{\omega^{T} P \omega} \leq \sqrt{\frac{\zeta \delta}{\vartheta}}\left\{\begin{array}{lll}
\|x\|_{\mathbf{d}}^{\nu+\alpha} & \text { if } & \|x\|_{\mathbf{d}} \leq 1, \\
\|x\|_{\mathbf{d}}^{\nu+\beta} & \text { if } & \|x\|_{\mathbf{d}} \geq 1,
\end{array}\right.
$$

where $\alpha$ and $\beta$ are defined in Theorem 2, then the inequality (12) of Theorem 6 holds.

In the case when the matrix $G_{\mathbf{d}}$ is diagonal, one can use another result presented in [7, Proposition 16] for simplification of the restriction (12). It is also worth to stress that the conditions (12), (14) can be considered locally if the operation domain is known a-priori.

\section{EXAMPLES}

\section{A. Finite-time stabilization of linear MIMO system (disturbance-free case)}

Consider the system (6) in disturbance-free case $(\tilde{d}(t, x)=$ 0 ) for $n=3$,

$$
A=\left(\begin{array}{ccc}
2 & 0 & 1 \\
-1 & 2 & 0 \\
2 & 0 & 1
\end{array}\right) \quad \text { and } \quad B=\left(\begin{array}{ll}
1 & 1 \\
0 & 1 \\
0 & 2
\end{array}\right)
$$

Define the finite-time control $u$ in the form (8) with the parameter $\nu=-0.3$, where the matrices $P \in \mathbb{R}^{3 \times 3}, K_{0}, K \in \mathbb{R}^{2 \times 3}$ are obtained from the inequalities (9), (10) with the parameters $\delta=1, \eta=0.8381$ :

$$
\begin{aligned}
P & =\left(\begin{array}{ccc}
0.0462 & -0.0572 & 0.0268 \\
-0.0572 & 0.1865 & -0.0593 \\
0.0268 & -0.0593 & 0.0622
\end{array}\right), \\
K_{0} & =\left(\begin{array}{ccc}
-2.9473 & 1.9473 & -0.9868 \\
-1.1053 & 0.1053 & -0.5263
\end{array}\right), \\
K & =\left(\begin{array}{ccc}
-4.2663 & 11.1510 & -3.6490 \\
0.7151 & -2.0240 & -0.4931
\end{array}\right), \\
G_{\mathbf{d}} & =\left(\begin{array}{ccc}
0.0972 & 0.3079 & -0.1539 \\
0 & 0.4130 & -0.1579 \\
0 & 0.0316 & 0.0814
\end{array}\right)
\end{aligned}
$$

and $\gamma=4142.2, \epsilon=1242.7$.

The numerical simulation of the closed-loop system has been done for $x_{0}=\left(\begin{array}{lll}-1 & 2 & 4\end{array}\right)^{T}$ by the Euler method with the fixed step size $h=0.005$. To find values of $\|\cdot\|_{\mathbf{d}}$ the bisection method was used. The results of simulation are shown in Fig. 1, Fig. 2.

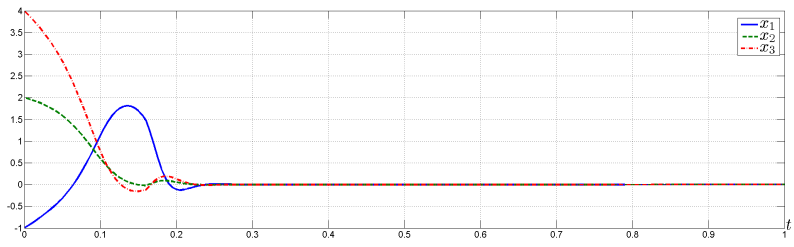

Fig. 1. System states versus time for finite-time control in disturbance-free case

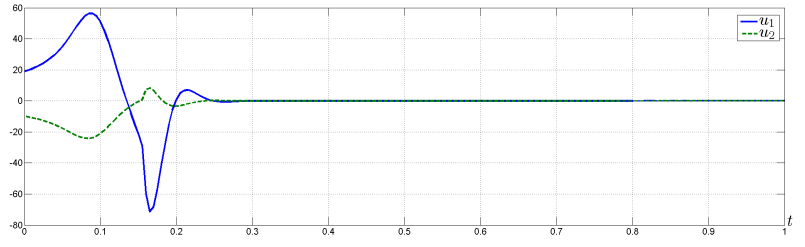

Fig. 2. Control inputs versus time

\section{B. Finite-time stabilization of linear MIMO system (disturbed} case)

Consider the same system in presence of disturbances $\omega_{1}(t, x(t))=\omega_{2}(t, x(t))=0, \omega_{3}(t, x(t))=1.5 \sin \left(x_{1}(t)\right)$. Define the finite-time control $u$ in the form (8) with the parameter $\nu=-0.1$, where the matrices $P \in \mathbb{R}^{3 \times 3}, K_{0}, K \in \mathbb{R}^{2 \times 3}$ are obtained from (9), (10b) - (11) with $\vartheta=30.9939, \delta=1$ :

$$
\begin{gathered}
P=10^{-1}\left(\begin{array}{ccc}
0.883 & -1.509 & 0.632 \\
-1.509 & 4.459 & -1.656 \\
0.632 & -1.656 & 1.062
\end{array}\right), \\
G_{\mathbf{d}}=10^{-2}\left(\begin{array}{ccc}
3.24 & 10.26 & -5.13 \\
0 & 13.77 & -5.26 \\
0 & 1.05 & 2.71
\end{array}\right), \\
K_{0}=\left(\begin{array}{ccc}
-2.9473 & 1.9473 & -0.9868 \\
-1.1053 & 0.1053 & -0.5263
\end{array}\right), \\
K=\left(\begin{array}{ccc}
-20.3529 & 38.6277 & -19.692 \\
7.2388 & -14.5646 & 3.3043
\end{array}\right),
\end{gathered}
$$


$\gamma=4142.2, \epsilon=414.2485$. According to Theorem 2 the condition (14) can be rewritten as follows

$$
\sqrt{\omega^{T} P \omega} \leq \sqrt{\frac{\zeta \delta}{\vartheta}}\left\{\begin{array}{lll}
\|x\|^{\frac{\nu+\alpha}{\beta}} & \text { if } & \|x\| \leq 1, \\
\|x\|^{\frac{\nu+\beta}{\alpha}} & \text { if } & \|x\| \geq 1 .
\end{array}\right.
$$

Then it can be easily checked that (14) holds with $\zeta=0.89$. The results of simulation are shown in Fig. 3, Fig. 4.

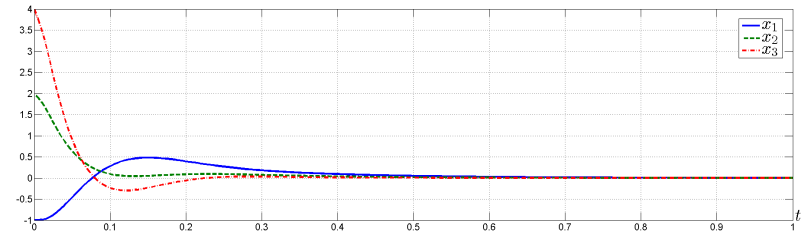

Fig. 3. System states versus time for finite-time control in disturbed case

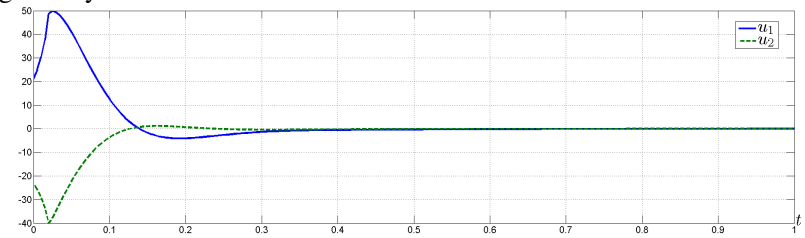

Fig. 4. Control inputs versus time

\section{Control of Twin Rotor MIMO System}

Obtained result has been implemented in practice on the TRMS 33-220 platform (see Fig. 5). In accordance with [28] the TRMS model can be represented in the form (6) with

$$
\begin{gathered}
x=\left(\begin{array}{lccccccc}
\psi & \dot{\psi} & \tau_{1} & \phi & \dot{\phi} & \tau_{2} & M_{R}
\end{array}\right)^{T}, \\
A=\left(\begin{array}{cccccccc}
0 & 1 & 0 & 0 & 0 & 0 & 0 \\
0 & -\frac{B_{1 \psi}}{l_{1}} & \frac{b_{1}}{l_{1}} & 0 & 0 & 0 & 0 \\
0 & 0 & -\frac{T_{10}}{T_{11}} & 0 & 0 & 0 & 0 \\
0 & 0 & 0 & 0 & 1 & 0 & 0 \\
0 & 0 & 0 & 0 & -\frac{B_{1 \phi}}{l_{2}} & \frac{b_{2}}{l_{2}} & -\frac{1}{l_{2}} \\
0 & 0 & 0 & 0 & 0 & -\frac{T_{20}}{T_{21}} & 0 \\
0 & 0 & \frac{k_{c}\left(-T_{0} T_{10}+T_{11}\right)}{T_{p} T_{11}} & 0 & 0 & 0 & -\frac{1}{T_{p}}
\end{array}\right) \\
B=\left(\begin{array}{ccccccccc}
0 & 0 & \frac{k_{1}}{T_{11}} & 0 & 0 & 0 & \frac{k_{c} T_{0} k_{1}}{T_{p} T_{11}} \\
0 & 0 & 0 & 0 & 0 & \frac{k_{2}}{T_{21}} & 0 & 0
\end{array}\right)^{T}, \\
\omega(t, x(t))=\left(\begin{array}{llllllll}
0 & \omega_{1} & 0 & 0 & \omega_{2} & 0 & 0
\end{array}\right)^{T},
\end{gathered}
$$

where $\psi$ is the pitch angle, $\phi$ is the yaw angle, $\tau_{1}, \tau_{2}$ are the vertical and horizontal motor moments, $M_{R}$ is the cross reaction momentum, $u_{i} \in[-2.5,2.5] \mathrm{V}, i=1,2$ are the control signals, $\omega_{1}=a_{1} x_{3}^{2}-M_{g} \sin x_{1}-B_{2 \psi} \operatorname{sign} x_{2}-$ $K_{g y}\left(a_{1} x_{3}^{2}+b_{1} x_{3}\right) x_{5} \cos x_{1}, \omega_{2}=a_{2} x_{6}^{2}-B_{2 \phi} \operatorname{sign} x_{5}$. The control law is chosen in the form $u(x)=u_{s s}+u_{n l}(x)$, where $u_{n l}=\left(\begin{array}{ll}u_{1} & u_{2}\end{array}\right)^{T}$ is the control law to be designed and $u_{s s}$ is static characteristic, which compensates gravity and crossreaction terms in steady state. All descriptions and values of model parameters are given in [28].

The parameters of the control $u_{n l}$ in the form (8) were selected solving the system (9), (10b)-(11) with $\nu=-0.8$, $\delta=0.3$ :

$$
K_{0}=\left(\begin{array}{ccccccc}
0 & 0.0011 & 1.6742 & 0 & 0 & 0 & 0.5309 \\
0 & -0.0448 & -17.8688 & 0 & -6.9441 & 7.4997 & -82.043
\end{array}\right) \text {, }
$$

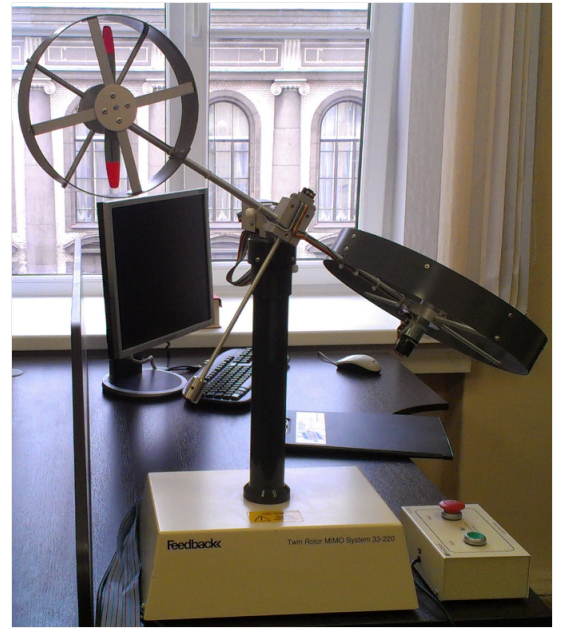

Fig. 5. Twin Rotor MIMO System

$$
\begin{aligned}
K & =\left(\begin{array}{ccccccc}
-61 & -210.5 & -151.1 & -50.7 & -70.2 & 4.9 & 134.9 \\
156.9 & 786.4 & 479.8 & -85.8 & -27 & -228.4 & 1403.3
\end{array}\right), \\
P & =10^{-2}\left(\begin{array}{ccccccc}
1.52 & 4.54 & 2.45 & -0.09 & 0.11 & -0.72 & 5.99 \\
4.54 & 22.66 & 12.84 & -1.44 & -0.01 & -3.6 & 33.19 \\
2.45 & 12.84 & 8.14 & -0.9 & -0.02 & -2.12 & 20.69 \\
-0.09 & -1.44 & -0.9 & 1.15 & 0.48 & 0.37 & -3.38 \\
0.11 & -0.01 & -0.02 & 0.48 & 0.82 & 0.12 & -1.08 \\
-0.72 & -3.6 & -2.12 & 0.37 & 0.12 & 1.01 & -5.98 \\
5.99 & 33.19 & 20.69 & -3.38 & -1.08 & -5.98 & 61.62
\end{array}\right), \\
G_{\mathbf{d}} & =\left(\begin{array}{ccccccc}
49.165 & -1.924 & -1.63 & 0 & 0 & 0 & -4.656 \\
-0.001 & 48.535 & 0.815 & 0 & 0 & 0 & 2.328 \\
-0 & 0.05 & 46.526 & 0 & -0.001 & 0.001 & -0.712 \\
-12.089 & 29.552 & 25.037 & 48.369 & 0 & 0 & 71.534 \\
0 & -14.697 & -12.518 & -0.001 & 47.569 & 0 & -35.767 \\
0.002 & -17.005 & -9.144 & -0.001 & 0.879 & 46.775 & -26.127 \\
0 & -0.087 & 0.302 & 0 & -0.001 & 0.001 & 47.637
\end{array}\right)
\end{aligned}
$$

and $\gamma=1.3241, \epsilon=47.8346$.

It can be seen the inequality (12) holds for $\|x\|_{\mathbf{d}} \geq \varrho, \varrho \in$ $\mathbb{R}_{+}$(i.e. the set $\left\{x \in \mathbb{R}^{n}:\|x\|_{\mathbf{d}} \leq \varrho\right\}$ is positively invariant).

Experimental results are presented in Fig. 6, Fig. 7. The measured signals $\psi, \phi$ were passed through low-pass filters. The experiment has been done with sampling period of the digital controller $h=0.001$. Peaks of control signal in steadystate are due to quantization of measurements of the signals.

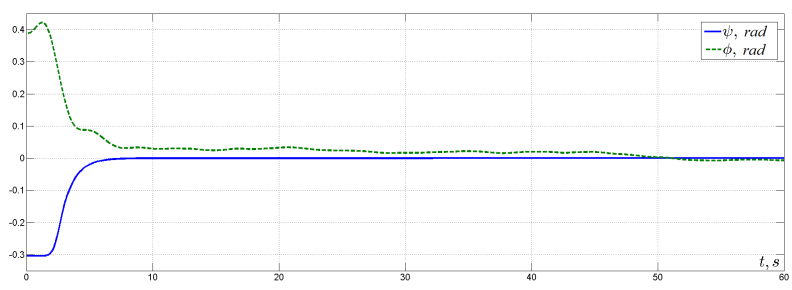

Fig. 6. Pitch angle $\psi$ and yaw angle $\phi$

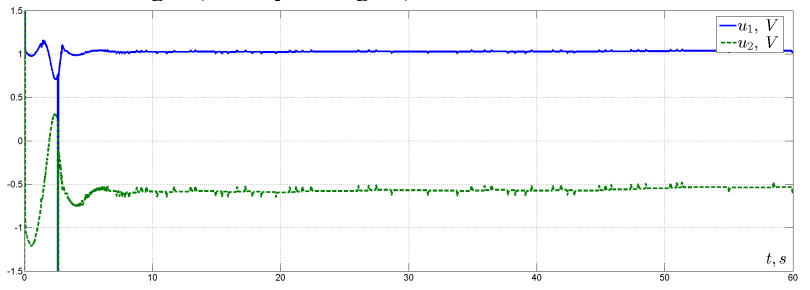

Fig. 7. Control signals for vertical and horizontal motors 


\section{CONCLUSION}

The paper presents homogenizing and stabilizing control algorithms for linear MIMO systems. It is shown that the system (6) can be homogenized with any degree via linear feedback. The presented stabilizing control law guarantees finite-time stability (asymptotic stability with a fixed-time attraction of any compact set containing the origin) if homogeneity degree is negative (positive). The settling time estimates are obtained. Tuning control parameters is presented in the form of linear matrix equations and inequalities. The robustness of the control algorithm with respect to system uncertainties and disturbances is studied.

\section{APPENDIX}

Proof of Lemma 2 Let $J \in \mathbb{R}^{n \times n}$ be the Jordan normal form of $X \in \mathbb{R}^{n \times n}$ obtained by similarity transformation $J=$ $T^{-1} X T$. Rewrite (5) in the form

$$
J \tilde{A}-\tilde{A} J=J,
$$

where $\tilde{A}=T^{-1} \bar{A} T$. If $X$ is nilpotent, then the matrix $J$ has nonzero elements only on its superdiagonal. Then the equation (15) always has a solution $\tilde{A} \in \mathbb{R}^{n \times n}$ (in diagonal form). Thus, for given nilpotent $X \in \mathbb{R}^{n \times n}$ the matrix equation (5) always has a solution $\bar{A} \in \mathbb{R}^{n \times n}$, e.g. if $J=\left(\begin{array}{lll}0 & 1 & 0 \\ 0 & 0 & 1 \\ 0 & 0 & 0\end{array}\right)$ then $\tilde{A}=\left(\begin{array}{lll}1 & 0 & 0 \\ 0 & 2 & 0 \\ 0 & 0 & 3\end{array}\right)$.

Proof of Lemma 3 Sufficiency. The system $\dot{x}=C x$ is d-homogeneous of degree $\nu$ if the identity (3) holds, i.e. $C e^{G_{\mathbf{d}} s}=e^{\nu s} e^{G_{\mathbf{d}} s} C=e^{\left(G_{\mathbf{d}}+\nu I_{n}\right) s} C$. Rewriting this expression as

$$
C \sum_{i=0}^{+\infty} \frac{s^{i} G_{\mathbf{d}}^{i}}{i !}=\sum_{i=0}^{+\infty} \frac{s^{i}\left(G_{\mathbf{d}}+\nu I_{n}\right)^{i}}{i !} C, \quad s \in \mathbb{R}
$$

and combining the terms of the same power we obtain that as sufficient condition for $\mathbf{d}$-homogeneity is that

$$
C G_{\mathbf{d}}^{i}=\left(G_{\mathbf{d}}+\nu I_{n}\right)^{i} C
$$

for any nonnegative integer $i$. For $i=0$ the equality is obvious. For $i=1$ it coincides with the condition (7). Since

$$
\begin{gathered}
C G_{\mathbf{d}}^{i+1}=C G_{\mathbf{d}} G_{\mathbf{d}}^{i}=\left(G_{\mathbf{d}}+\nu I_{n}\right) C G_{\mathbf{d}}^{i}= \\
\left(G_{\mathbf{d}}+\nu I_{n}\right)^{2} C G_{\mathbf{d}}^{i-1}=\cdots=\left(G_{\mathbf{d}}+\nu I_{n}\right)^{i} C G_{\mathbf{d}}= \\
\left(G_{\mathbf{d}}+\nu I_{n}\right)^{i+1} C,
\end{gathered}
$$

then by induction the equality (16) holds for all $i \geq 0$.

Necessity. Let $M(s)=C e^{G_{\mathbf{d}} s}-e^{\left(G_{\mathbf{d}}+\nu I_{n}\right) s} C$. Then for d-homogeneous system $\dot{x}=C x$ of degree $\nu$ due to (3) we have $M(s)=0$ and

$$
\frac{\partial M(s)}{\partial s}=C G_{\mathbf{d}} e^{G_{\mathbf{d}} s}-\left(G_{\mathbf{d}}+\nu I_{n}\right) e^{\left(G_{\mathbf{d}}+\nu I_{n}\right) s} C=0, \forall s \in \mathbb{R} .
$$

Finally, taking $s=0$ we obtain that the condition (7) holds.

Proof of Theorem 4 Sufficiency. According to Lemma 3, the system (6), $u=K_{0} x, \omega(t, x)=0$ is d-homogeneous of degree $\nu$ if and only if

$$
\left(A+B K_{0}\right) G_{\mathbf{d}}-G_{\mathbf{d}}\left(A+B K_{0}\right)=\nu\left(A+B K_{0}\right) .
$$

Let the matrix $K_{0}$ is chosen such that the matrix $A+B K_{0} \in$ $\mathbb{R}^{n \times n}$ is nilpotent. Then, according to Lemma 2 , there is a matrix $L \in \mathbb{R}^{n \times n}$ that is a solution of the Sylvester equation $\left(A+B K_{0}\right) L-L\left(A+B K_{0}\right)=A+B K_{0}$. The matrix $\bar{L} \in$ $\mathbb{R}^{n \times n}, \bar{L}=L+\frac{\epsilon}{\nu} I_{n}$ is also its solution for any $\epsilon \in \mathbb{R}_{+}$, i.e. $\left(A+B K_{0}\right)\left(L+\frac{\epsilon}{\nu} I_{n}\right)-\left(L+\frac{\epsilon}{\nu} I_{n}\right)\left(A+B K_{0}\right)=(A+$ $\left.B K_{0}\right) L-L\left(A+B K_{0}\right)$. Finally, choosing the parameter $\epsilon$ for the generator in the form $G_{\mathbf{d}}=\nu L+\epsilon I_{n}$ to be anti-Hurwiz and substituting the generator matrix into the equation (17) complete the sufficiency proof.

Necessity. Let the equality (17) hold. Rewrite (17) in the form (5) as $\left(A+B K_{0}\right) \bar{G}_{\mathbf{d}}-\bar{G}_{\mathbf{d}}\left(A+B K_{0}\right)=A+B K_{0}$, where $\bar{G}_{\mathbf{d}}=\frac{1}{\nu} G_{\mathbf{d}}$. Then, according to Lemma 1, any matrix solution $A+B K_{0} \in \mathbb{R}^{n \times n}$ is nilpotent.

\section{Proof of Lemma 4 Let}

$$
u(x)=K_{0} x+\tilde{u}(x)
$$

be a d-homogeneously stabilizing feedback with degree $\nu \in$ $\mathbb{R}$, the generator matrix is $G_{\mathbf{d}} \in \mathbb{R}^{n \times n}$ and $K_{0}$ is such that (17) is satisfied. Then for $\mathbf{d}$-homogeneous term $\tilde{u}(x)$ we have

$$
B \tilde{u}(\mathbf{d}(s) x)=e^{\nu s} \mathbf{d}(s) B \tilde{u}(x) .
$$

Note that according to Theorem 2 there exists a matrix $P$ satisfying (2). Let us consider the new dilation $\breve{\mathbf{d}}(s)=e^{\breve{G}_{\mathbf{d}} s}$ where $\breve{G}_{\mathbf{d}}=\rho I_{n}-G_{\mathbf{d}}$ and $\rho \in \mathbb{R}_{+}$is such that (2) is satisfied with corresponding generator $\breve{G}_{\mathbf{d}}$ and the same matrix $P$. Note that $\left(A+B K_{0}\right) x$ is $\mathbf{d}$-homogeneous with degree $-\nu$ due to

$\left(A+B K_{0}\right) \breve{G}_{\mathbf{d}}-\breve{G}_{\mathbf{d}}\left(A+B K_{0}\right)=G_{\mathbf{d}}\left(A+B K_{0}\right)-\left(A+B K_{0}\right) G_{\mathbf{d}}$.

Then the system (6) with the control

$$
\breve{u}(x)=K_{0} x+\|x\|_{\breve{\mathbf{d}}}^{\rho} \tilde{u}\left(\frac{x}{\|x\|_{\breve{\mathbf{d}}}^{\rho}}\right)
$$

is $\breve{\mathbf{d}}$-homogeneous with degree $-\nu$ since taking into account (19) we have

$$
\begin{aligned}
& \|\breve{\mathbf{d}}(s) x\|_{\breve{\mathbf{d}}}^{\rho} B \tilde{u}\left(\frac{\breve{\mathbf{d}}(s) x}{\|\breve{\mathbf{d}}(s) x\|_{\breve{d}}^{\rho}}\right)=e^{\rho s}\|x\|_{\breve{\mathbf{d}}}^{\rho} B \tilde{u}\left(\frac{e^{\rho s} \mathbf{d}(-s) x}{e^{\rho s}\|x\|_{\breve{\mathbf{d}}}^{\rho}}\right)= \\
& e^{(\rho-\nu) s}\|x\|_{\breve{\mathbf{d}}}^{\rho} \mathbf{d}(-s) B \tilde{u}\left(\frac{x}{\|x\|_{\breve{\mathbf{d}}}^{\rho}}\right)=e^{-\nu s}\|x\|_{\breve{\mathbf{d}}}^{\rho} \breve{\mathbf{d}}(s) B \tilde{u}\left(\frac{x}{\|x\|_{\breve{\mathbf{d}}}^{\rho}}\right) .
\end{aligned}
$$

For $\|x\|_{\breve{\mathbf{d}}} \leq 1$ and $y=\breve{\mathbf{d}}\left(-\ln \|x\|_{\breve{\mathbf{d}}}\right) x$ (i.e. $y \in\left\{x:\|x\|_{\breve{d}}=\right.$ $1\}=S$ ) we have

$$
\begin{gathered}
\|\| x\left\|_{\breve{\mathbf{d}}}^{\rho} B \tilde{u}\left(\frac{x}{\|x\|_{\breve{d}}^{\rho}}\right)\right\|=\|\| x\left\|_{\breve{\mathbf{d}}}^{\rho} B \tilde{u}\left(\frac{\breve{\mathbf{d}}\left(\ln \|x\|_{\breve{\mathbf{d}}}\right) y}{\|x\|_{\breve{\mathbf{d}}}^{\rho}}\right)\right\|= \\
\|\| x\left\|_{\breve{\mathbf{d}}}^{\rho-\nu} \mathbf{d}\left(-\ln \|x\|_{\breve{\mathbf{d}}}\right) B \tilde{u}(y)\right\| \leq\|x\|_{\breve{\mathbf{d}}}^{\rho-\nu-\alpha}\|B \tilde{u}(y)\|,
\end{gathered}
$$

where $\alpha$ is defined in Theorem 2 , then $\breve{u}(x) \rightarrow 0$ as $x \rightarrow 0$ for sufficiently large $\rho \in \mathbb{R}_{+}(\rho>\nu+\alpha)$ and the control $\breve{u}(x)$ is continuous on $\mathbb{R}^{n} \backslash\{0\}$ if $\tilde{u}(x)$ is continuous on the unit sphere $S$. The stability of the system (6), (20) is straightforward since the systems (6), (20) and (6), (18) coincide on the unit sphere $S$ and the Lyapunov function can be chosen the same as for the system (6), (18). Finally, according to [15] the system (6), (18) (system (6), (20)) is $\mathbf{d}^{\varsigma}$-homogeneous ( $\mathbf{d}^{\varsigma}$-homogeneous) with degree $\varsigma \nu(-\varsigma \nu)$ for any $\varsigma \in \mathbb{R}_{+}$with $\mathbf{d}^{\varsigma}(s)=\mathbf{d}(\varsigma s)$ 
$\left(\breve{\mathbf{d}^{\varsigma}}(s)=\breve{\mathbf{d}}(\varsigma s)\right)$ and the generator $G_{\mathbf{d}^{\varsigma}}=\varsigma G_{\mathbf{d}}\left(G_{\breve{\mathbf{d}}^{\varsigma}}=\varsigma G_{\breve{\mathbf{d}}}\right)$.

Proof of Theorem 5 and Lemma 5 Let us present the proof of Theorem 5, where sufficiency part coincides with the proof of Lemma 5.

Necessity. If we assume that the linear disturbance free system (6) is $\mathbf{d}$-homogeneously stabilizable with degree $\nu \neq 0$ then according to Lemma 4 it is $\mathbf{d}$-homogeneously stabilizable with any degree. In particular, it is finite-time stablizable for $\nu<0$. The equivalence of controllability and finite-time stabilizability of linear dynamical systems is well known (see, for example, [29]).

Sufficiency. Let us prove sufficiency in two steps: first we show that the feedback law (8) stabilizes the system (6) if (9) - (10) hold, and then feasibility of (9) - (10) is proven in the case of controllable pair $\{A, B\}$.

I. Let $\nu \geq-1$. Choose the candidate Lyapunov function $V=\|x\|_{\mathbf{d}}$. Since $\|x\|_{\mathbf{d}}=e^{s}:\|\mathbf{d}(-s) x\|=1$,

$$
\begin{gathered}
\frac{\partial\|\mathbf{d}(-s) x\|}{\partial s}=-\left.\frac{\partial\|z\|}{\partial z}\right|_{z=\mathbf{d}(-s) x} G_{\mathbf{d}} \mathbf{d}(-s) x= \\
-\frac{1}{2}\|\mathbf{d}(-s) x\|^{-1} x^{T} \mathbf{d}^{T}(-s)\left(P G_{\mathbf{d}}+G_{\mathbf{d}}^{T} P\right) \mathbf{d}(-s) x, \\
\frac{\partial\|\mathbf{d}(-s) x\|}{\partial x}=\|\mathbf{d}(-s) x\|^{-1} x^{T} \mathbf{d}^{T}(-s) P \mathbf{d}(-s),
\end{gathered}
$$

then implying $\frac{\partial s}{\partial x}=-\left[\frac{\partial\|\mathbf{d}(-s) x\|}{\partial s}\right]^{-1} \frac{\partial\|\mathbf{d}(-s) x\|}{\partial x}$ (by means of Implicit Function Theorem [30] for the function $s: \mathbb{R}^{n} \rightarrow \mathbb{R}$ implicitly defined by $\|\mathbf{d}(-s) x\|=1)$ we obtain

$$
\begin{gathered}
\dot{V}=\frac{\partial}{\partial t}\|x\|_{\mathbf{d}}=\frac{\partial}{\partial x}\|x\|_{\mathbf{d}} \dot{x}(t)=\left.e^{s} \frac{\partial s}{\partial x}\right|_{s=\ln \|x\|_{\mathbf{d}}} \times \\
\left(A x+B K_{0} x+\|x\|_{\mathbf{d}}^{\nu(1+\gamma)+\epsilon} B K \mathbf{d}\left(-\ln \|x\|_{\mathbf{d}}\right) x\right)= \\
\left(x^{T} \mathbf{d}^{T}\left(-\ln \|x\|_{\mathbf{d}}\right)\left(P G_{\mathbf{d}}+G_{\mathbf{d}}^{T} P\right) \mathbf{d}\left(-\ln \|x\|_{\mathbf{d}}\right) x\right)^{-1} \times \\
2\|x\|_{\mathbf{d}} x^{T} \mathbf{d}^{T}\left(-\ln \|x\|_{\mathbf{d}}\right) P \mathbf{d}\left(-\ln \|x\|_{\mathbf{d}}\right) \times \\
\left(A x+B K_{0} x+\|x\|_{\mathbf{d}}^{\nu(1+\gamma)+\epsilon} B K \mathbf{d}\left(-\ln \|x\|_{\mathbf{d}}\right) x\right) .
\end{gathered}
$$

Taking into account (9b), the equation (9a) is equivalent to $\left(A+B K_{0}\right) L-L\left(A+B K_{0}\right)=A+B K_{0}$ with $K_{0}=$ $y_{0}\left(L-(\gamma+1) I_{n}\right)^{-1}$. According to (9c) the matrix $L-(\gamma+1) I_{n}$ is invertible. Since the equation $\left(A+B K_{0}\right) L-L\left(A+B K_{0}\right)=$ $A+B K_{0}$ holds, then in accordance with Theorem 4 we have $A x+B K_{0} x$ is d-homogeneous of degree $\nu$ with the generator $G_{\mathbf{d}}=\nu L+\epsilon I_{n}$ (the inequality (10c) imply that the matrix $G_{\mathbf{d}}$ is anti-Hurwiz):

$$
\mathbf{d}(-s)\left(A+B K_{0}\right) x=e^{\nu s}\left(A+B K_{0}\right) \mathbf{d}(-s) x .
$$

The inequality (10c) guarantees that

$$
\eta P \geq P G_{\mathbf{d}}+G_{\mathbf{d}}^{T} P>0
$$

and according to Theorem 2 the dilation $\mathbf{d}$ is strictly monotone.

Let us show that the equality

$$
\begin{gathered}
\mathbf{d}\left(-\ln \|x\|_{\mathbf{d}}\right)\|x\|_{\mathbf{d}}^{\nu(1+\gamma)+\epsilon} B K \mathbf{d}\left(-\ln \|x\|_{\mathbf{d}}\right) x= \\
\|x\|_{\mathbf{d}}^{\nu} B K \mathbf{d}\left(-\ln \|x\|_{\mathbf{d}}\right) x .
\end{gathered}
$$

holds. To do this, it enough to show that

$$
\left(e^{(\nu \gamma+\epsilon) s} \mathbf{d}(-s)-I\right) B K=0 .
$$

Since

$$
\left(e^{(\nu \gamma+\epsilon) s} \mathbf{d}(-s)-I\right) B K=\sum_{i=1}^{+\infty} \frac{s^{i}\left((\nu \gamma+\epsilon) I_{n}-G_{\mathbf{d}}\right)^{i}}{i !} B K
$$

the equality (25) holds if $\left(G_{\mathbf{d}}-(\nu \gamma+\epsilon) I_{n}\right) B K=0$ which is implied by (9b). Then, using (25) it is easy to show that (24) holds.

Returning to (21) with using (10a), (22), (23), (24) we obtain

$$
\begin{gathered}
\dot{V}=\frac{\|x\|_{\mathbf{d}}^{1+\nu} z^{T}\left(P\left(A+B K_{0}+B K\right)+\left(A+B K_{0}+B K\right)^{T} P\right) z}{z^{T}\left(P G_{\mathbf{d}}+G_{\mathbf{d}}^{T} P\right) z} \leq \\
-\frac{\delta\|x\|_{\mathbf{d}}^{1+\nu} z^{T} P z}{\eta z^{T} P z}=-\frac{\delta}{\eta} V^{1+\nu} .
\end{gathered}
$$

where $z=\mathbf{d}\left(-\ln \|x\|_{\mathbf{d}}\right) x$.

In the case $\nu<-1$ choose the candidate Lyapunov function in the form $\tilde{V}=V^{\mu}=\|x\|_{\mathbf{d}}^{\mu}$, where $\mu \geq-\nu$. Then according to (26) we can obtain

$$
\dot{\tilde{V}} \leq-\frac{\mu \delta}{\eta} \tilde{V}^{\frac{\mu+\nu}{\mu}}
$$

Finally, applying Theorem 1 for (26) (or (27)) shows that the feedback law (8) stabilizes the system (6) with different types of convergence if (9) - (10) hold.

II. Let us show that the system of equations and inequalities (9)-(10) is feasible if the pair $\{A, B\}$ is controllable.

To show that the equations (9) are feasible let us use the known block decomposition procedure discussed in [7], [23]. Due to this reason all decomposition descriptions and details are skipped and only resulting system is shown:

$$
\dot{s}=\tilde{A} s+\tilde{B} \tilde{u},
$$

where $s \in \mathbb{R}^{n}$ is a new variable after transformation, $\tilde{u}$ is a control input, $\tilde{A} \in \mathbb{R}^{n \times n}, \tilde{B} \in \mathbb{R}^{n \times m}$. The matrices $\tilde{A}, \tilde{B}$ have the following block forms

$$
\begin{gathered}
\tilde{A}=\left(\begin{array}{ccccc}
0 & A_{12} & 0 & \ldots & 0 \\
0 & 0 & A_{23} & \ldots & 0 \\
\ldots & \ldots & \cdots & \ldots & \ldots \\
0 & 0 & \ldots & 0 & A_{k-1 k} \\
A_{k 1} & A_{k 2} & \ldots & A_{k k-1} & A_{k k}
\end{array}\right) \\
\tilde{B}=\left(\begin{array}{lllll}
0 & 0 & \ldots & 0 & A_{k k+1}^{T}
\end{array}\right)^{T}
\end{gathered}
$$

where $k$ is the number of blocks (in both rows and columns), $A_{i j} \in \mathbb{R}^{n_{i} \times n_{j}}, i, j=1,2, \ldots, k, n_{1}+n_{2}+\cdots+n_{k}=n$, $\operatorname{rank}\left(A_{i i+1}\right)=n_{i}, A_{k k+1} \in \mathbb{R}^{m \times m}, n_{k}=m$. Choose $\tilde{u}$ as linear control input

$$
\tilde{u}=\tilde{K}_{0} s=-A_{k k+1}^{+}\left(\begin{array}{lllll}
A_{k 1} & A_{k 2} \quad \cdots \quad A_{k k-1} \quad A_{k k}
\end{array}\right) s,
$$

where $A_{k k+1}^{+}$is the left inverse matrix to $A_{k k+1}$. Then

$$
\dot{s}=\left(\tilde{A}+\tilde{B} \tilde{K}_{0}\right) s=\left(\begin{array}{ccccc}
0 & A_{12} & 0 & \ldots & 0 \\
0 & 0 & A_{23} & \ldots & 0 \\
\ldots & \ldots & \ldots & \ldots & \ldots \\
0 & 0 & \ldots & 0 & A_{k-1 k} \\
0 & 0 & \ldots & 0 & 0
\end{array}\right) s .
$$

Returning to (9) with corresponding transformed matrices $\tilde{A}, \tilde{B}, \tilde{K}_{0}$ it can be easily shown that $\tilde{L}=$ 
$\left(\begin{array}{cccc}(\gamma-k+1) I_{n_{1}} & 0 & \cdots & 0 \\ 0 & (\gamma-k+2) I_{n_{2}} & \cdots & 0 \\ \cdots & \cdots & \cdots & \cdots \\ 0 & \cdots & \gamma I_{n_{k}}\end{array}\right)$ is an solution of the equa-

tions (9) with any $\gamma \in \mathbb{R}$ and the matrix

$\tilde{L}-(\gamma+1) I_{n}=\left(\begin{array}{cccc}-k I_{n_{1}} & 0 & \cdots & 0 \\ 0 & (-k+1) I_{n_{2}} & \ldots & 0 \\ \ldots & \ldots & \ldots & \ldots \\ 0 & \ldots & 0 & -I_{n_{k}}\end{array}\right)<0$

is negative and invertible (i.e. the inequality (9c) is satisfied). Thus, (9) are always feasible for controllable pair $\{A, B\}$.

Controllability of the pair $\{A, B\}$ implies feasibility of the matrix inequalities (10a), (10b) for any given $K_{0} \in \mathbb{R}^{m \times n}$ (see, for example, [31]). Finally, the inequality (10c) is feasible for sufficiently big $\epsilon$ and $\eta$.

Proof of Theorem 6 Let us take the same candidate Lyapunov functions as in thre proof of Theorem 5. Taking into account the disturbances we obtain

$$
\begin{gathered}
\frac{\partial\left\|\mathbf{d}\left(-\ln \|x\|_{\mathbf{d}}\right)\right\|}{\partial x}\left(A x+B K_{0} x+\|x\|_{\mathbf{d}}^{\nu+\epsilon} B K \mathbf{d}\left(-\ln \|x\|_{\mathbf{d}}\right) x+\omega\right)= \\
\frac{1}{2}\|x\|_{\mathbf{d}}^{\nu} x^{T} \mathbf{d}^{T}\left(-\ln \|x\|_{\mathbf{d}}\right)\left(P\left(A+B K_{0}+B K\right)+\right. \\
\left.\left(A+B K_{0}+B K\right)^{T} P\right) \mathbf{d}\left(-\ln \|x\|_{\mathbf{d}}\right) x+ \\
x^{T} \mathbf{d}^{T}\left(-\ln \|x\|_{\mathbf{d}}\right) P \mathbf{d}\left(-\ln \|x\|_{\mathbf{d}}\right) \omega= \\
\frac{1}{2}\left(\begin{array}{c}
\mathbf{d}\left(-\ln \|x\|_{\mathbf{d}}\right) x \\
\mathbf{d}\left(-\ln \|x\|_{\mathbf{d}}\right) \omega
\end{array}\right)^{T} W\left(\begin{array}{l}
\mathbf{d}\left(-\ln \|x\|_{\mathbf{d}}\right) x \\
\mathbf{d}\left(-\ln \|x\|_{\mathbf{d}}\right) \omega
\end{array}\right)- \\
\frac{1}{2} \delta\|x\|_{\mathbf{d}}^{\nu} x^{T} \mathbf{d}^{T}\left(-\ln \|x\|_{\mathbf{d}}\right) P \mathbf{d}\left(-\ln \|x\|_{\mathbf{d}}\right) x+ \\
\frac{1}{2}\|x\|_{\mathbf{d}}^{-\nu} \omega^{T} \mathbf{d}^{T}\left(-\ln \|x\|_{\mathbf{d}}\right) R^{-1} \mathbf{d}\left(-\ln \|x\|_{\mathbf{d}}\right) \omega= \\
\frac{1}{2}\left(\begin{array}{l}
\mathbf{d}\left(-\ln \|x\|_{\mathbf{d}}\right) x \\
\mathbf{d}\left(-\ln \|x\|_{\mathbf{d}}\right) \omega
\end{array}\right)^{T} W\left(\begin{array}{l}
\mathbf{d}\left(-\ln \|x\|_{\mathbf{d}}\right) x \\
\mathbf{d}\left(-\ln \|x\|_{\mathbf{d}}\right) \omega
\end{array}\right)- \\
\frac{1}{2} \delta\|x\|_{\mathbf{d}}^{\nu}+\frac{1}{2}\|x\|_{\mathbf{d}}^{-\nu} \omega^{T} \mathbf{d}^{T}\left(-\ln \|x\|_{\mathbf{d}}\right) R^{-1} \mathbf{d}\left(-\ln \|x\|_{\mathbf{d}}\right) \omega,
\end{gathered}
$$

where

$W=\left(\begin{array}{cc}\|x\|_{\mathbf{d}}^{\nu}\left(P\left(A+B K_{0}+B K\right)+\left(A+B K_{0}+B K\right)^{T} P+\delta P\right) & P \\ P & -\|x\|_{\mathbf{d}}^{-\nu} R^{-1}\end{array}\right)$

The matrix $W$ is negative semidefinite (it can be obtained from (11) by using Schur complement taking into account $P=X^{-1}$ and $K=y P$ ). Then the inequality (12) implies $\dot{V} \leq-\frac{(1-\zeta) \delta}{\eta} V^{1+\nu}$ for the case $\nu \geq-1\left(\dot{\tilde{V}} \leq-\frac{\mu(1-\zeta) \delta}{\eta} V^{\frac{\mu+\nu}{\mu}}\right.$ for the case $\left.\nu<-1\right)$.

Proof of Corollary 1 The inequality (13) implies $R^{-1} \leq$ $\vartheta P$. According to [6, the proof of Corollary 4], the inequality

$$
\begin{aligned}
& \omega^{T} \mathbf{d}^{T}\left(-\ln \|x\|_{\mathbf{d}}\right) P \mathbf{d}\left(-\ln \|x\|_{\mathbf{d}}\right) \omega \leq \\
& \omega^{T} P \omega\left\{\begin{array}{lll}
\|x\|_{\mathbf{d}}^{-2 \alpha} & \text { if } \quad\|x\|_{\mathbf{d}} \leq 1 \\
\|x\|_{\mathbf{d}}^{-2 \beta} & \text { if } \quad\|x\|_{\mathbf{d}} \geq 1
\end{array}\right.
\end{aligned}
$$

holds. Then, utilizing these inequalities one can rewrite the inequality (12) as sufficient condition in the form (14).

\section{REFERENCES}

[1] K. Zimenko, A. Polyakov, D. Efimov and W. Perruquetti, Generalized Feedback Homogenization and Stabilization of Linear MIMO Systems, European Control Conference (ECC), pp. 1987-1991, 2018.

[2] M. Kawski, Geometric Homogeneity and Stabilization, in Proc. IFAC Nonlinear Control Symposium, A. Krener and D. Mayne, Eds., Lake Tahoe, CA, pp. 164-169, 1995.

[3] L. Rosier, Etude de Quelques Problemes de Stabilization [PhD thesis], Paris, France: Ecole Normale Superieure de Cachan, 1993.
[4] V. Khomenuk, On systems of ordinary differential equations with generalized homogeneous right-hand sides, Izvestia vuzov. Mathematica, vol. 3(22), pp. 157-164, 1961 (in Russian).

[5] A. Polyakov, D. Efimov, E. Fridman and W. Perruquetti, On Homogeneous Distributed Parameter Systems, IEEE Transactions on Automatic Control, vol. 61(11), pp. 3657-3662, 2016.

[6] A. Polyakov, Sliding Mode Control Design Using Canonical Homogeneous Norm, Int. J. Robust. Nonlinear Control, pp. 1-20, DOI: 10.1002/rnc.4058, 2018

[7] A. Polyakov, D. Efimov and W. Perruquetti, Robust Stabilization of MIMO Systems in Finite/Fixed Time, Int. J. Robust. Nonlinear Control, vol. 26(1), pp. 69-90, 2016.

[8] S. Bhat and D. Bernstein, Finite-time stability of continuous autonomous systems, SIAM Journal of Control and Optimization, vol. 38(3), pp. 751-766, 2000.

[9] Y. Orlov, Finite Time Stability and Robust Control Synthesis of Uncertain Switched Systems, SIAM Journal of Control and Optimization, vol. 43(4), pp. 1253-1271, 2004.

[10] S. Laghrouche, M. Harmouche and Y. Chitour, Stabilization of perturbed integrator chains using Lyapunov-Based Homogeneous Controllers, International Journal of Control, pp. 1-10, 2016.

[11] A. Polyakov, J.-M. Coron and L. Rosier, On finite-time stabilization of evolution equations: A homogeneous approach, in Proceedings of 55th Conference on Decision and Control (CDC), pp. 3143-3148, 2016.

[12] E. Bernuau, D. Efimov and W. Perruquetti, Robustness of Homogeneous and Homogeneizable Differential Inclusions, In: Li S., Yu X., Fridman L., Man Z., Wang X. (eds) Advances in Variable Structure Systems and Sliding Mode Control - Theory and Applications. Studies in Systems, Decision and Control, vol. 115, Springer, Cham, pp. 39-56, 2018.

[13] E. Bernuau, A. Polyakov, D. Efimov and W. Perruquetti, Verification of ISS, iISS and IOSS properties applying weighted homogeneity, System and Control Letters, vol. 62, no. 12, pp. 1159-1167, 2013.

[14] E. Ryan, Universal stabilization of a class of nonlinear systems with homogeneous vector fields, Systems \& Control Letters, vol. 26, pp. 177-184, 1995.

[15] A. Polyakov, Quadratic-like Stability of Nonlinear Homogeneous Systems, 56th IEEE Conference on Decision and Control, Melbourne, Australia, Dec 2017

[16] E. Moulay and W. Perruquetti, Finite-time stability and stabilization: State of the art, Lecture Notes in Control and Information Sciences, vol. 334, pp. 23-41, 2006

[17] V. Zubov, On systems of ordinary differential equations with generalized homogenous right-hand sides, Izvestia vuzov. Mathematica, vol. 1, pp. 80-88, 1958 (in Russian).

[18] S.P. Bhat, D.S. Bernstein, Geometric homogeneity with applications to finite-time stability, Mathematics of Control, Signals and Systems, vol. 17(2), pp. 101-127, 2005.

[19] A. Levant, Homogeneity approach to high-order sliding mode design, Automatica, vol. 41, pp. 823-830, 2005.

[20] V. Zubov, Methods of A.M. Lyapunov and Their Applications, Noordhoff, Leiden, 1964

[21] A. Bacciotti and L. Rosier, Lyapunov Functions and Stability in Control Theory, Springer, 2005.

[22] D. Efimov, A. Polyakov, W. Perruquetti and J.-P. Richard, Weighted homogeneity for time-delay systems: Finite-time and independent of delay stability, IEEE Trans. Automatic Control, vol. 61(1), pp. 210-215, 2016.

[23] A. Polyakov, Nonlinear Feedback Design for Fixed-Time Stabilization of Linear Control Systems, IEEE Transactions on Automatic Control, vol. 57(8), pp. 2106-2110, 2012.

[24] A. Pazy, Semigroups of Linear Operators and Applications to Partial Differential Equations, Springer, 1983

[25] D. Burde, On the Matrix Equation $X A-A X=X^{P}$, Linear Algebra and its Applications vol. 404, pp. 147-165, 2005.

[26] F.R. Gantmacher, The theory of matrices, AMS Chelsea Publishing, Providence, RI, 1998.

[27] A.F. Filippov, Differential equations with discontinuous right-hand sides, Kluwer, Dordrecht, 1988.

[28] Twin Rotor MIMO System, East Sussex, UK: Feedback Instruments Ltd, 2006.

[29] Y. Hong, Finite-time stabilization and stabilizability of a class of controllable systems, Systems \& Control Letters, vol. 46(4), pp. 231236, 2002.

[30] R. Courant and F. John, Introduction to Calculus and Analysis, vol. II/1, New York: Springer, 2000.

[31] S. Boyd, E. Ghaoui, E. Feron and V. Balakrishnan, Linear Matrix Inequalities in System and Control Theory, Philadelphia: SIAM, 1994. 